\title{
Évaluation de deux méthodes acoustiques de détermination du module d'élasticité de bois de mélèze hybride jeune (Larix $\times$ eurolepis Henry) - comparaison avec une méthode normalisée en flexion statique
}

\author{
Michèle Marchal*, Dominique Jacques** \\ Centre de recherche de la nature, des forêts et du bois, ministère de la région Wallonne, DGRNE, avenue Maréchal-Juin, \\ 23, B-5030 Gembloux, Belgique
}

(Reçu le 21 juillet 1998, accepté le 21 janvier 1999)

\begin{abstract}
Evaluation of two acoustic methods of MOE determination for young hybrid larch wood (Larix $\times$ eurolepis Henry). Comparison with a standard method by static bending. Based on 384 normalised specimens sampled in 32 hybrid larch stems from a 14-year-old clonal test, wood quality was evaluated by measuring the modulus of elasticity by means of two acoustic testing methods, the major point of interest being the speed of handling. Results were compared to those given by a third method, chosen as the reference one and described by the French norm NF B 51-016. Furthermore, specific gravity and ring width were measured and presence of compression wood noted. Simple regression equations gave close relationships between each of the two acoustic methods and the reference one. This could lead to interesting applications in the field of large scale systematic tests. Finally, the impact of some factors (clone, ramet in clone, compression wood, density and ring width) were evaluated by the residue distribution around the regression line. (C) Inra/Elsevier, Paris)
\end{abstract}

non destructive method / modulus of elasticity / scatter analysis / compression wood / clone / Larix $\times$ eurolepis Henry

Résumé - Sur la base de 384 éprouvettes normalisées $(20 \times 20 \times 360 \mathrm{~mm})$, issues de 32 arbres d'un test clonal de 14 ans de mélèze hybride, le module d'élasticité a été déterminé à l'aide de deux méthodes acoustiques dont l'intérêt majeur est la rapidité d'exécution. L'une de ces méthodes est basée sur la mesure du temps de propagation d'une onde ultrasonore dans le sens longitudinal et l'autre, sur la fréquence de résonance propre. Les résultats ont été comparés à ceux obtenus à l'aide d'une troisième méthode choisie comme référence et décrite par la norme française NF B 51-016. Outre le module d'élasticité, la masse volumique et la largeur des cernes ont été mesurées, et la présence de bois de réaction a été notée. Les équations de régression simple établies sur la base des données fournies par les deux méthodes alternatives et la méthode de référence font apparaître des relations étroites autorisant des perspectives intéressantes en matière de tests systématiques à grande échelle. Enfin, l'impact de différents facteurs (clone, ramet dans clone, bois de compression, largeur des cernes, masse volumique) sur la distribution des résidus autour des droites de régression a été étudié. (ㅇ Inra/Elsevier, Paris)

méthode non destructive / module d'élasticité / analyse de la dispersion / bois de compression / clone / Larix $\times$ eurolepis Henry

\footnotetext{
*Correspondance et tirés à part: Mi.Marchal@mrw.wallonie.be
}

**D.Jacques@marw.wallonie.be 


\section{Introduction}

Dans le cadre d'un programme d'amélioration génétique, le test systématique des caractéristiques mécaniques du bois à l'échelle d'une population de base nécessite des moyens considérables en temps et maind'œuvre. La mise au point de techniques de mesure plus rapides et applicables à du matériel jeune pourrait rendre possible la détermination systématique de cette caractéristique mécanique, en plus d'autres critères habituellement pris en compte, tels que la croissance et la forme.

L'évaluation de la rigidité du bois se pratique traditionnellement, en laboratoire, par la mesure du module d'élasticité en flexion statique sur éprouvettes standardisées soumises à des tests normalisés. Cette méthode est lente et laborieuse et nécessite un conditionnement particulier du matériau étudié (débit sur quartier vrai, choix de bois exempt de défaut,...).

Depuis les années 80 , des chercheurs tentent d'utiliser d'autres techniques basées sur des principes permettant des mesures plus rapides, techniques qui, apparemment, conduisent à des résultats satisfaisants $[3,18]$. La littérature disponible concerne principalement les genres Abies, Cryptomeria, Picea et Pinus testés sous forme d'éprouvettes sans défaut, mais aussi, de rondins, bois de construction ou lamellés-collés [5, 10, 11, 13, 21, 23].

Le but de cet article est, d'une part, de comparer les valeurs du module d'élasticité estimées par deux méthodes acoustiques aux valeurs obtenues par la méthode normalisée de détermination de la flexion statique d'éprouvettes standardisées décrite par la norme française NF B 51-016 [2] et, d'autre part, d'étudier la dispersion de ces données autour des droites de régression en relation avec différents facteurs susceptibles d'influencer la qualité de ces relations.

\section{Matériel et méthodes}

\subsection{Matériel expérimental}

Le matériel utilisé est issu d'une plantation âgée de 14 ans d'une variété multiclonale ${ }^{1}$ de mélèze hybride (Larix $\times$ eurolepis Henry), fruit du croisement entre le mélèze d'Europe (Larix decidua Mill) et le mélèze du Japon (Larix kaempferi (Lamb) Carr). Seize clones représentés par deux ramets ${ }^{2}$, soit 32 arbres, forment le matériel de base dans lequel un ensemble de 384 éprou-

\footnotetext{
${ }^{1}$ Mélange de plants bouturés issus de la sélection de plusieurs clones particulièrement performants ; un clone étant un ensemble de plants génétiquement identiques.

${ }^{2}$ Copie végétative d'un plant issu de semis.
}

vettes ont été découpées dans l'aubier. Le prélèvement des éprouvettes, de dimensions $20 \times 20 \times 360 \mathrm{~mm}$, a été effectué conformément à la norme NF B 51-016 [2]: découpe sur quartier, dans le sens longitudinal, de bois exempt de défauts. Il faut cependant constater que, dans des arbres aussi jeunes, il n'a pas toujours été possible de prélever des éprouvettes exemptes de tout bois de compression et qu'un nombre non négligeable de ces éprouvettes portent les traces de ce défaut.

Notons enfin qu'un taux d'humidité d'équilibre de $12 \%$ par rapport à la masse anhydre a été maintenu tout au long des différents essais.

\subsection{Mode de prélèvement des éprouvettes}

Le fût a été débité, à partir de la base, en 3 billons (de pied, intermédiaire, supérieur) de longueur comprise entre 1,5 à 2,5 m. Dans chacun d'eux, quatre plateaux ont été découpés suivant les orientations nord-est, sudest, sud-ouest et nord-ouest ; un barreau de longueur variable a été prélevé dans l'aubier de chacun de ces plateaux. Enfin, après avoir ramené le taux d'humidité à $12 \%$ par un séjour en enceinte climatisée, les barreaux ont été amenés par découpe et rabotage aux dimensions d'éprouvettes d'essais de $20 \times 20 \times 360 \mathrm{~mm}$.

\subsection{Méthodes d'essais}

\subsubsection{Méthode de référence}

La méthode, décrite par la norme française NF B 51016 [2], permet de déterminer le module d'élasticité dans la zone de flexion pure en déformation élastique, d'une éprouvette soumise à une charge progressive croissante exercée perpendiculairement au fil du bois, parallèlement aux cernes (figure l).

Le module d'Young dans le sens longitudinal $\left(\mathrm{MOE}_{4 \mathrm{PT}}\right)$, exprimé en mégapascals (MPa), est donné par l'équation :

$$
\mathrm{MOE}_{4 \mathrm{PT}}=\frac{3 \mathrm{P}(\mathrm{l}-\mathrm{a}) \mathrm{m}^{2}}{8 \mathrm{bh}^{3} \mathrm{f}}
$$

dans laquelle : $\mathrm{P}=$ charge totale appliquée, en newtons (N) ;

$1=$ distance entre les axes des appuis cylindriques, en millimètres $(\mathrm{mm})$;

$a=$ distance entre les axes des têtes de chargement $(\mathrm{mm})$;

$\mathrm{m}=$ distance séparant les points de contact du support de l'instrument de mesure de la flèche $(\mathrm{mm})$; 
$\mathrm{h}=$ hauteur de l'éprouvette au milieu de sa longueur, parallèle aux cernes $(\mathrm{mm})$;

$\mathrm{b}=$ largeur de l'éprouvette au milieu de la longueur, perpendiculaire aux cernes $(\mathrm{mm})$;

$\mathrm{f}=$ flèche dans la zone de flexion pure $(\mathrm{mm})$.

\subsubsection{Méthode de la fréquence de résonance à l'aide du Grindo-Sonic ${ }^{3}$}

La mesure d'une pièce de matière quelconque par le Grindo-Sonic se base sur la mesure de phénomènes vibratoires dissipant l'énergie générée par le choc entre cette pièce et un objet quelconque [24].

Ces phénomènes vibratoires sont complexes et dépendent de la nature de la matière, de la force de l'impact ainsi que des caractéristiques physiques du corps mis en vibration [1, 6, 7]. L'amortissement des vibrations, variable d'une matière à l'autre, peut, dès lors, être une caractéristique de la nature de cette matière.

L'appareil Grindo-Sonic transforme le signal reçu de cette fréquence naturelle en courant électrique de même fréquence et de même amplitude relative, sur une durée de huit périodes, à l'aide d'une horloge à quartz dont le cristal de référence oscille à $2 \mathrm{MHz}$ [19] (figure 2).

La lecture effectuée correspond à la durée de deux périodes propres ; elle est exprimée en $\mu \mathrm{s}$.

La fréquence propre ( $\mathrm{Fr}$ - herz) est donnée par :

$$
F_{r}=\frac{2 \times 10^{6}}{R}
$$

dans laquelle $: R=$ durée de deux périodes propres $(\mu \mathrm{s})$.

Le module dynamique caractéristique $\left(\mathrm{MOE}_{\mathrm{GR}}-\right.$ $\mathrm{MPa}$ ) est donné par l'équation transformée de Spinner and Tefft [24] appliquée à des éprouvettes de section transversale rectangulaire :

$$
\mathrm{MOE}_{\mathrm{GR}}=3,78568 \frac{\mathrm{ML}^{3}}{\mathrm{~b} \mathrm{~h}^{3} \mathrm{R}^{2}} 10^{6}
$$

dans laquelle : $\mathbf{M}=$ masse, en grammes $(\mathrm{g})$;

$\mathrm{L}=$ longueur $(\mathrm{mm})$

$\mathrm{b}=$ base $(\mathrm{mm})$;

$\mathrm{h}=$ hauteur $(\mathrm{mm})$.

Le choc initial a été appliqué perpendiculairement à l'éprouvette et tangentiellement aux cernes, la sonde étant radiale (figure 2).

\footnotetext{
${ }^{3}$ Mis au point par JW Lemmens, Dynamic Materials Testing Instruments ; Geldenaaksebaan, 456, B-3001 Leuven, Belgique, Modèle MK3S.
}

\subsubsection{Méthode ultrasonore à l'aide du Sylvatest ${ }^{4}$}

Le Sylvatest permet de mesurer le temps de propagation d'une onde ultrasonore (fréquence d'émission $50 \mathrm{kHz}$ ) dans le sens longitudinal de l'éprouvette (figure 3).

La vitesse de propagation de l'onde ultrasonore dans le bois $\left(\mathrm{V}_{\mathrm{US}}-\mathrm{m} \mathrm{s}^{-1}\right)$, quotient de la longueur de l'échantillon par le temps mesuré, est utilisée pour estimer les valeurs du module d'élasticité $[4,5,10,22]$ selon l'équation spécifique :

$$
\mathrm{MOE}_{\mathrm{US}}=\mathrm{V}_{\mathrm{US}}^{2} 10^{-6}=\frac{\mathrm{ML}}{\mathrm{bh} \mathrm{t}^{2}} 10^{-6}
$$

dans laquelle : $\mathrm{V}_{\mathrm{US}}=$ vitesse de l'ultrason dans le sens longitudinal, en mètres par seconde $\left(\mathrm{m} \mathrm{s}^{-1}\right)$;

$\rho=$ masse volumique de l'éprouvette, en kilogrammes par mètre cube $\left(\mathrm{kg} \mathrm{m}^{-3}\right)$;

$\mathrm{t}=$ temps de traversée de l'onde ultrasonore $(\mu \mathrm{s})$;

$\mathrm{M}, \mathrm{L}, \mathrm{b}, \mathrm{h}=$ définis au paragraphe 2.3.2 ;

$\mathrm{MOE}_{\mathrm{US}}=$ module d'élasticité (constante de rigidité) dans le sens longitudinal (MPa).

Il est à signaler que, travaillant avec des éprouvettes de $360 \mathrm{~mm}$, nous nous trouvons en dessous de la limite de $500 \mathrm{~mm}$ préconisée par le constructeur, ce qui risque d'affecter légèrement la précision des mesures.

\subsection{Mesures complémentaires des caractéristiques du bois}

Différentes caractéristiques du bois réputées influencer le module d'élasticité, comme la présence de bois de compression, la masse volumique, la largeur des cernes, ont été évaluées.

\subsubsection{Bois de compression}

Le bois de compression, présent $(\mathrm{BC}=1)$ ou absent $(\mathrm{BC}=0)$, a été évalué sur la base de la coloration du bois. Sa présence est considérée comme significative si l'on perçoit cette coloration sur les deux côtés de l'éprouvette en coupe radiale.

\subsubsection{Masse volumique}

La masse volumique $\left(\rho-\mathrm{kg} \mathrm{m}^{-3}\right)$ est une caractéristique essentielle, intégrée dans l'estimation du module par les deux méthodes alternatives. Elle a été calculée

\footnotetext{
${ }^{4}$ Mis au point par JL Sandoz, École polytechnique fédérale de Lausanne, IBOIS Construction en Bois ; $\mathrm{GCH} 2$ Ecublens, $\mathrm{CH}-$ 1015 Lausanne, Suisse.
} 


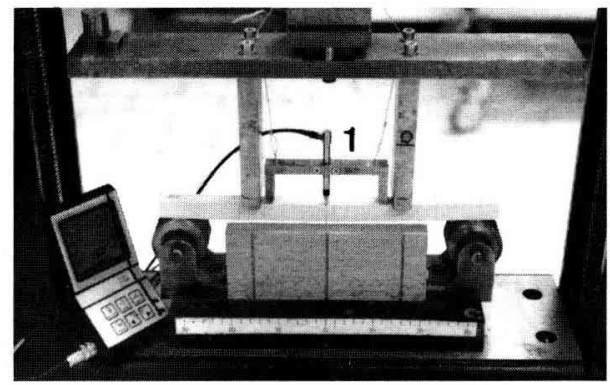

Figure 1. Détermination du module d'élasticité en flexion statique à quatre points d'appuis : mesure de la flèche $(\mu \mathrm{m})$ à l'aide d'un capteur de déplacement (1).

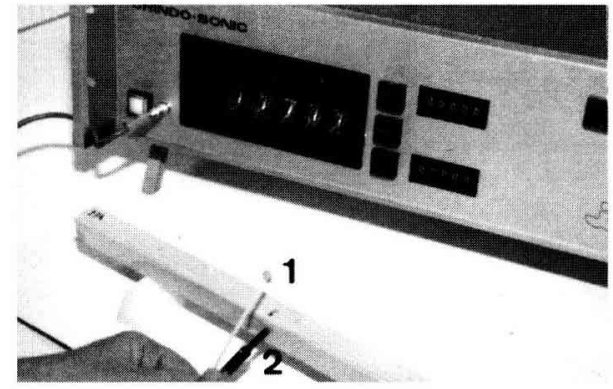

Figure 2. Mesure de la fréquence de résonance à l'aide de l'appareil Grindo-Sonic : point d'impact (1) et détecteur de vibrations propres (2).

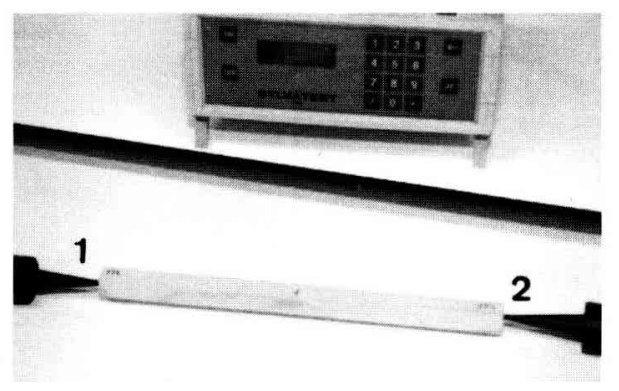

Figure 3. Mesure de la vitesse de propagation de l'onde ultrasonore dans le sens longitudinal à l'aide de l'appareil Sylvatest : sonde émettrice (1), sonde réceptrice (2).

sur la base de la mesure des dimensions des barreaux, par méthode gravimétrique.

\subsubsection{Largeur des cernes}

La largeur des cernes ( $\mathrm{mm}$ ) est définie comme le quotient de deux fois la largeur moyenne de l'éprouvette (mm) par le nombre total de cernes apparaissant sur les deux faces transversales.

\section{Résultats et discussion}

\subsection{Comparaison des estimations du module d'élasticité obtenues par les deux méthodes acoustiques par rapport à la méthode de référence}

Les tableaux $I, I I$ et $I I I$, ainsi que la figure 4, présentent les résultats globaux de cette étude. Par souci de clarté, les deux méthodes acoustiques seront évaluées séparément.

\subsubsection{Cas de la mesure de la fréquence de résonance}

Le tableau I met en évidence une sous-estimation moyenne de près de $8 \%$ du module d'élasticité mesuré à l'aide de la fréquence de résonance, par rapport à la méthode de référence ; la sous-estimation observée est confirmée et même renforcée pour les maxima (12\%), alors que les minima sont pratiquement égaux.

La figure 4 confirme cette observation. D'un biais moyen de moins de $5 \%$ constaté pour de faibles modules d'élasticité (5000 MPa), nous passons, pour les mesures voisines de $12000 \mathrm{MPa}$, à un biais de l'ordre de $10 \%$. Ces valeurs diffèrent des résultats présentés par Haines et al. [14] qui font apparaître une sous-estimation moyenne de l'ordre de 0,4 et $2,3 \%$ pour Picea abies et Abies alba, cet ordre de grandeur étant confirmé par Haines et Leban [15] sur Picea abies. En revanche, Görlacher [12] observe une surestimation de l'ordre de $8 \%$ sur Pseudotsuga menziesii.

Dans notre cas, il n'est donc pas envisageable d'utiliser cette méthode telle quelle. Etant donné la forme de la 
Tableau I. Valeurs du module d'élasticité (MPa) déterminé par la méthode de référence (MOE $\left.\mathrm{MPT}_{\mathrm{PT}}\right)$ et les deux méthodes acoustiques : la mesure de la fréquence de résonance $\left(\mathrm{MOE}_{\mathrm{GR}}\right)$ et la mesure de la vitesse de l'onde ultrasonore (MOE $\left.\mathrm{MS}_{\mathrm{U}}\right)$; valeurs de la masse volumique $\left(\mathrm{kg} \mathrm{m}^{-3}\right)$ et de la largeur des cernes $(\mathrm{mm})$ pour un ensemble de 384 éprouvettes de mélèze hybride.

\begin{tabular}{ccccc}
\hline & Moyenne & Minimum & Maximum & Écart-type \\
\hline Module (MPa) MOE $_{4 \mathrm{PT}}$ & 8700 & 3700 & 14600 & 1900 \\
MOE $_{\mathrm{GR}}$ & 8000 & 3700 & 12900 & 1700 \\
MOE $_{\mathrm{US}}$ & 10000 & 5600 & 16000 & 1800 \\
Masse volumique $\left(\mathrm{kg} \mathrm{m}^{-3}\right)$ & 416 & 351 & 525 & 31 \\
Largeur des cernes $(\mathrm{mm})$ & 7,3 & 4,0 & 13,3 & 1,5 \\
\hline
\end{tabular}

Tableau II. Équations de régression calculées sur la base des valeurs du module d'élasticité mesuré par la méthode de référence et par les deux méthodes acoustiques (fréquence de résonance et vitesse de l'onde ultrasonore) pour un ensemble de 384 éprouvettes de mélèze hybride.

\begin{tabular}{lcccc}
\hline Méthode utilisée & $\mathrm{N}^{\circ}$ & Modèle et coefficients & Nombre d'éprouvettes & Coefficient de détermination \\
\hline Fréquence de résonance & 1 & $\mathrm{y}=1,145 \times-475$ & 384 & $\mathrm{R}^{2}=0,97 * * * 5$ \\
Vitesse de l'onde ultrasonore & 2 & $\mathrm{y}=0,975 \times-1082$ & 384 & $\mathrm{R}^{2}=0,87 * * *$ \\
\hline
\end{tabular}

Tableau III. Test de conformité et niveau de signification du coefficient angulaire $(a=1)$ et de l'ordonnée à l'origine $(b=0)$ des équations de régression calculées sur la base des valeurs du module d'élasticité mesuré par la méthode de référence et par les deux méthodes acoustiques (fréquence de résonance et vitesse de l'onde ultrasonore) pour un ensemble de 384 éprouvettes de mélèze hybride.

\begin{tabular}{lcc}
\hline $\begin{array}{l}\text { Méthode } \\
\text { utilisée }\end{array}$ & du coefficient angulaire $(\mathrm{a}=1)$ & $\begin{array}{c}\text { Niveau de signification } \\
\text { de l'ordonnée à l'origine }(\mathrm{b}=0)\end{array}$ \\
\hline $\begin{array}{l}\text { Fréquence de résonance } \\
\text { Vitesse de l'onde ultrasonore }\end{array}$ & $1,145 * * *$ & $-475 * * *$ \\
& $0,975 \mathrm{~ns}$ & $-1082 * * *$
\end{tabular}

distribution des points, le calcul d'une droite de régression apparaît opportun afin d'obtenir une relation simple et non biaisée dans la gamme des modules étudiés (tableau $I I$, équation 1 ). Le coefficient de détermination de la droite ainsi définie est très élevé $\left(R^{2}=0,97 * * *\right)$ et la dispersion des points autour de la droite apparaît très faible (figure 4).

Le test de conformité du coefficient angulaire [8] et de l'ordonnée à l'origine (logiciel SAS, procédure REG) confirme les observations faites sur la base graphique : le coefficient angulaire et l'ordonnée à l'origine apparaissent en effet bien différents, le premier de 1 et la seconde de 0 (tableau $I I I)$.

\footnotetext{
${ }^{5}$ Symboles utilisés pour l'ensemble du document. ns : non significatif ;

$*$ : significatif pour $\alpha=0,05$;

** : significatif pour $\alpha=0,01$;

$* * *$ : significatif pour $\alpha=0,001$.
}

\subsubsection{Cas de la mesure de la vitesse de l'onde ultrasonore}

Dans le cas de la méthode ultrasonore, contrairement aux données fournies par la fréquence de résonance, le tableau I fait apparaître une surestimation d'environ $15 \%$ par rapport à la méthode de référence, aussi bien pour la valeur moyenne que pour les minimum et maximum. La figure 4 met également en évidence cette surévaluation, de l'ordre de $1500 \mathrm{MPa}$, et montre l'apparente constance des écarts quelle que soit la gamme des modules mesurés. Ces résultats concordent avec ceux obtenus par Haines et Leban [15] qui observent, sur la base de 19 éprouvettes de Picea abies, une surestimation de $14 \%$. Dans une étude antérieure, Haines et al. [14] obtiennent une surestimation de 17 et $22 \%$ respectivement avec Picea abies et Abies alba.

Comme dans le cas de la fréquence de résonance, il s'avère donc indispensable de calculer une droite de régression d'allure simple pour évaluer le module d'élasticité de référence (tableau $I$, équation 2). La dispersion 


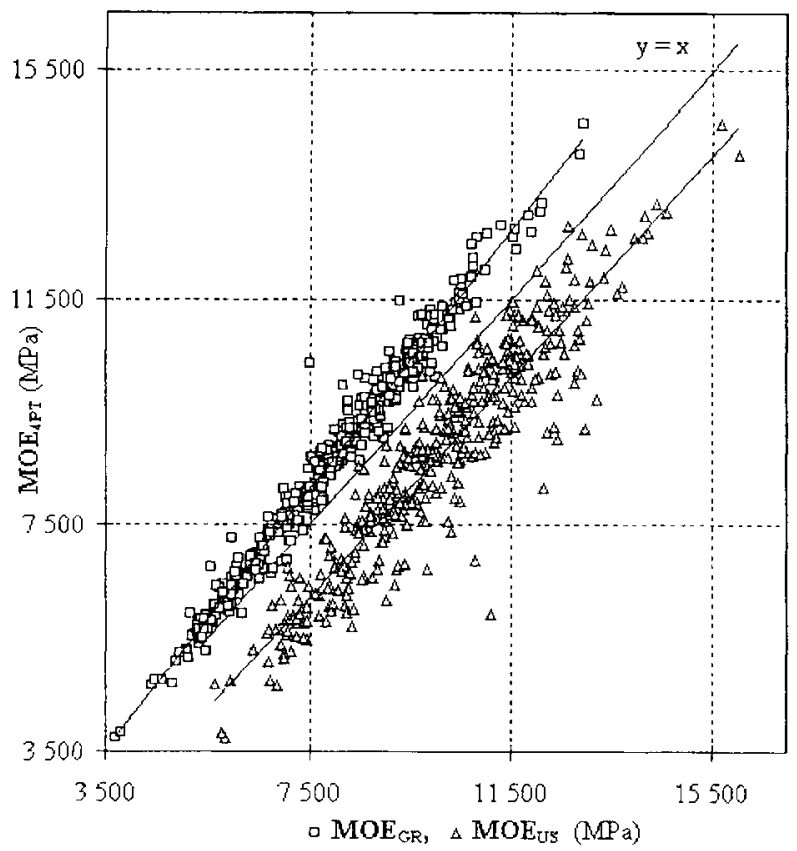

Figure 4. Relations entre les modules d'élasticité (MPa) mesurés par la méthode de référence $\left(\mathrm{MOE}_{4 \mathrm{PT}}\right)$, la méthode de la fréquence de résonance $\left(\mathrm{MOE}_{\mathrm{GR}}\right)$ et la méthode de la vitesse de l'onde ultrasonore $\left(\mathrm{MOE}_{\mathrm{US}}\right)$ pour l'échantillon complet $(n=384)$.

du nuage des points autour de la droite de régression est cependant plus importante que pour la première méthode $\left(R^{2}=0,87 * * *\right)$ comme le soulignent également Baiilleres et al. [3] sur base d'essais comparatifs réalisés sur deux essences tropicales ; par contre, le coefficient angulaire de la droite de régression simple calculée à partir de ce nuage de points n'est pas significativement différent de 1 (tableau III). Ces constatations contrastent avec celles de Bucur [4] sur 18 éprouvettes de Fagus sylvatica, qui, bien qu'obtenant un coefficient de corrélation très élevé $(R=0,98)$, met en évidence un coefficient angulaire très éloigné de $1(a=0,58)$.

\subsection{Impacts de différents facteurs et de quelques caractéristiques du bois sur la qualité des droites de régression obtenues}

Les régressions simples entre le module de référence et ceux déduits des deux méthodes acoustiques étant établies, et bien que la dispersion autour des droites soit relativement faible, il convient de vérifier l'influence que pourraient avoir sur la qualité de ces deux relations diffé- rents facteurs liés à la nature du matériel testé ainsi que différentes caractéristiques du bois. Ceci devrait permettre de préciser l'origine du biais éventuel que pourrait provoquer l'usage de l'une ou de l'autre de ces deux techniques par rapport aux mesures normalisées $\left(\mathrm{MOE}_{4 \mathrm{PT}}\right)$.

Pour ce faire, les résidus des droites de régression ont été calculés comme suit [9] :

$$
d_{i}=y_{i}-y\left(x_{i}\right)
$$

avec $d_{i}=$ résidu pour l'observation $\mathrm{i}$;

$y_{i}=$ valeur mesurée par la méthode de référence pour l'observation $\mathrm{i}$;

$y\left(x_{1}\right)=$ valeur calculée par la droite de régression pour l'observation $\mathrm{i}$.

Ces résidus sont soumis soit à une analyse de la variance à 1 ou deux facteurs dans le cas de facteurs qualitatifs (bois de compression, clone, ramet, billon, orientation), soit au calcul des coefficients de corrélation de Pearson dans le cas des variables continues (masse volumique, largeur de cerne). Les données sont traitées à l'aide du logiciel $\mathrm{SAS}^{6}$ (Procédures REG, GLM et CORR).

Les modèles d'analyse étant particuliers aux différents facteurs étudiés, ils seront détaillés par la suite.

\subsubsection{Présence de bois de compression}

Sachant que la présence de bois de compression peut avoir une influence très négative sur l'élasticité du matériau bois pour de nombreux résineux $[17,20,25]$, nous avons évalué l'impact de cette caractéristique au moyen d'une analyse de la variance à un critère de classification appliquée aux résidus des deux droites de régression calculées préalablement (tableau II, équations 1 et 2 ).

Le modèle s'établit comme suit :

$$
Y_{i j}=\mu+B_{i}+\varepsilon_{i j}
$$

avec $Y_{i j}=$ observation j du type de bois i ;

$\mu=$ moyenne générale ;

$B_{i}=$ effet du type de bois $(\mathrm{BC}=1$ ou $\mathrm{BC}=0$, facteur fixe) ;

$\varepsilon_{i j}=$ résidu lié au modèle d'analyse.

Dans ces conditions, nous constatons que la présence de bois de compression influence de manière très hautement significative les résidus des deux régressions testées (tableau IV).

En moyenne, en l'absence de bois de compression, les droites de régression calculées pour les deux méthodes

\footnotetext{
${ }^{6}$ System for Windows v6.12
} 
acoustiques sous-estiment le module d'élasticité (tableau $V$ ). Cette sous-estimation est cependant relativement faible : 38 et $154 \mathrm{MPa}$ respectivement pour la fréquence de résonance et la vitesse de l'onde ultrasonore.

En présence de ce défaut, nous observons, au contraire, une surestimation plus importante en valeur absolue et quatre fois plus importante dans le cas de la méthode ultrasonore que dans celui de la fréquence de résonance.

Pour la méthode ultrasonore, le calcul de droites de régression spécifiques à ces deux types d'échantillons, l'un contenant du bois de compression et l'autre en étant exempt, confirme cette constatation et aboutit à la définition de deux droites dont les coefficients angulaires sont significativement différents (figure 5).

Si, sur le plan théorique, la définition de ces deux droites se justifie pleinement, il faut cependant admettre qu'en pratique il est difficilement imaginable d'utiliser deux formules de régression, voire une droite de régression multiple, étant donné la difficulté d'évaluer de manière rigoureuse et rapide l'importance du bois de compression au niveau des éprouvettes.

Les deux droites de régression calculées pour les mesures de la fréquence de résonance en séparant les éprouvettes contenant du bois de compression de celles qui sont exemptes de ce défaut (figure 6, tableau VI) sont caractérisées toutes deux par une coefficient angulaire assez proche de 1 ainsi que par un terme indépendant proche de 0 . Ce tri permet ainsi de réduire fortement le biais introduit par cette technique spécialement en présence de bois de compression. Cependant, comme dans le cas de la vitesse de l'onde ultrasonore, il semble difficile de tenir compte de ces observations pour des applications pratiques.

\subsubsection{Facteurs clone et ramet dans clone}

Dans le cadre de la caractérisation des propriétés mécaniques de différents matériels génétiques, il est important de vérifier si les écarts entre les méthodes

Tableau IV. Influence de la présence/absence de bois de compression $(\mathrm{BC}=1 / 0)$ sur les résidus des droites de régression établies entre la méthode de référence et les deux méthodes acoustiques. Tableau simplifié d'analyse de la variance à un critère de classification.

\begin{tabular}{lccc}
\hline Méthode & Degré de liberté & Carré moyen & Valeur de F \\
\hline Fréquence de résonance & 1 & 1364267 & $14,02 * * *$ \\
Résidus & 382 & 97332 & $51,48 * * *$ \\
Vitesse de l'onde ultrasonore & 1 & 22637517 & 439744 \\
Résidus & 382 & 430 & \\
\hline
\end{tabular}

Tableau V. Résidus moyens observés (MPa) pour les deux méthodes acoustiques par rapport à la méthode de référence lors de la mesure du module d'élasticité, pour un ensemble de 384 éprouvettes dont 110 contiennent du bois de compression $(\mathrm{BC}=1)$ et 274 en sont exemptes $(\mathrm{BC}=0)$.

\begin{tabular}{lccc}
\hline Méthode & \multicolumn{2}{c}{ Résidus (MPa) } & $\begin{array}{c}\text { Écart moyen entre les deux types } \\
\text { d'éprouvettes (MPa) }\end{array}$ \\
\hline Fréquence de résonance & -94 & $B C=0$ & 132 \\
Vitesse de l'onde ultrasonore & -383 & 154 & 537 \\
\hline
\end{tabular}

Tableau VI. Équations de régression calculées sur base des valeurs du module d'élasticité mesuré par la méthode de référence et par les deux méthodes acoustiques (fréquence de résonance et vitesse de l'onde ultrasonore) pour un ensemble de 384 éprouvettes de mélèze hybride dont 110 contiennent du bois de compression $(B C=1)$ et 274 en sont exemptes $(B C=0)$.

\begin{tabular}{lcccc}
\hline Méthode utilisée & Bois de compression & Modèle et coefficients & Nombre d'éprouvettes & Coefficient de détermination \\
\hline Fréquence de & $\mathrm{BC}=1$ & $\mathrm{y}=1,088 \times-193$ & 110 & $\mathrm{R}^{2}=0,97 * * *$ \\
résonance & $\mathrm{BC}=0$ & $\mathrm{y}=1,123 \times-249$ & 274 & $\mathrm{R}^{2}=0,96 * * *$ \\
Vitesse de l'onde & $\mathrm{BC}=1$ & $\mathrm{y}=0,764 \times+342$ & 110 & $\mathrm{R}^{2}=0,78 * * *$ \\
ultrasonore & $\mathrm{BC}=0$ & $\mathrm{y}=0,936 \times-513$ & 274 & $\mathrm{R}^{2}=0,85 * * *$ \\
\hline
\end{tabular}




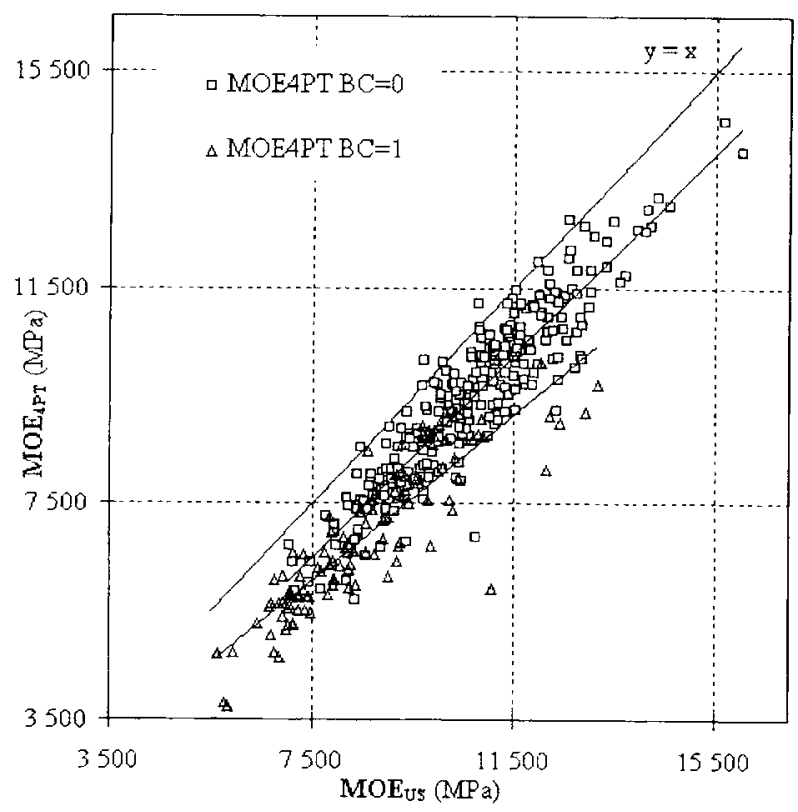

Figure 5. Relation entre les modules d'élasticité (MPa) établis par la méthode de référence $\left(\mathrm{MOE}_{4 \mathrm{PT}}\right)$ et la méthode ultrasonore $\left(\mathrm{MOE}_{\mathrm{US}}\right)$ pour un ensemble de 384 éprouvettes de mélèze hybride dont 110 contiennent du bois de compression $(\mathrm{BC}=1)$ et 274 en sont exemptes $(\mathrm{BC}=0)$.

acoustiques et la méthode de référence sont affectés ou non d'un effet systématique des pools génétiques soumis à comparaison.

Grâce au type d'échantillonnage réalisé, il est possible de contrôler la distribution des résidus au niveau interclonal (facteur clone) et intraclonal (facteur ramet dans clone) pour les deux méthodes acoustiques testées, en réalisant une analyse de la variance de type hiérarchisé à deux facteurs, définie par le modèle suivant :

$$
Y_{i j k}=C_{i}+R(C)_{i j}+\varepsilon_{i j k}
$$

avec $Y_{i j k}=$ observation $\mathrm{k} \mathrm{du}$ ramet $\mathrm{j}$ dans le clone $\mathrm{i}$;

$C_{i}=$ effet du clone $\mathrm{i}$ (facteur aléatoire) ;

$R(C)_{i j}=$ effet $\mathrm{du}$ ramet $\mathrm{j}$ dans le clone $\mathrm{i}$ (facteur aléatoire) ;

$\varepsilon_{i j k}=$ résidu lié au modèle d'analyse.

Pour l'échantillon total, les résultats de cette analyse ne permettent pas de mettre en évidence un effet génétique (clone) sur la distribution des résidus des deux régressions étudiées (tableau VII). L'effet intraclonal (ramet dans clone) est par contre au moins significatif pour le deux méthodes.

Quand l'analyse se base sur les éprouvettes exemptes de bois de compression ( $\mathrm{BC}=0 ; n=274$ ), tous ces

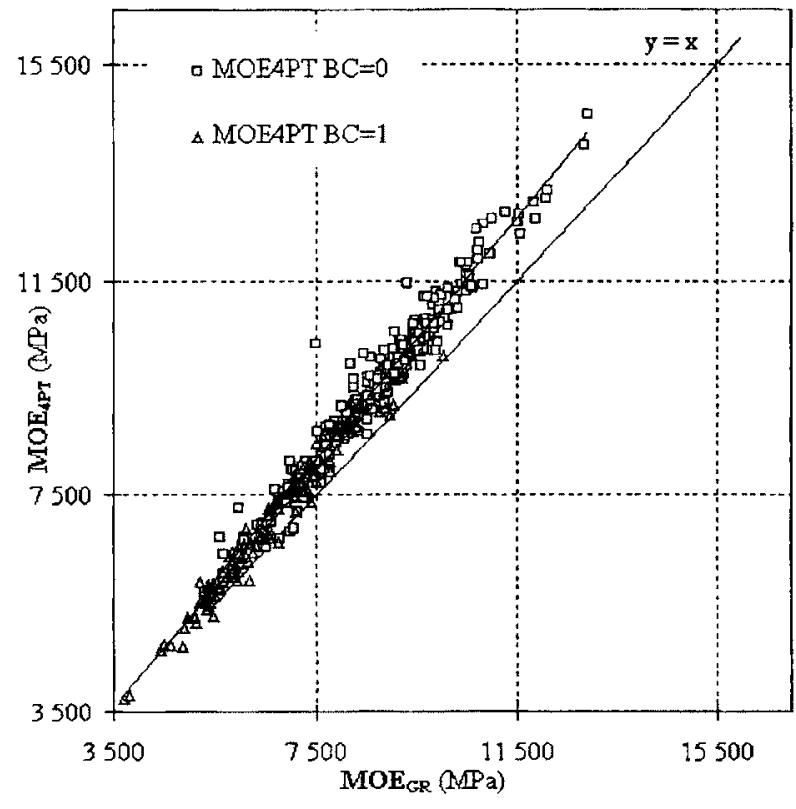

Figure 6. Relation entre les modules d'élasticité (MPa) établis par la méthode de référence $\left(\mathrm{MOE}_{4 \mathrm{PT}}\right.$ ) et la méthode de la fréquence de résonance $\left(\mathrm{MOE}_{\mathrm{GR}}\right)$ pour un ensemble de 384 éprouvettes de mélèze hybride dont 110 contiennent du bois de compression $(\mathrm{BC}=1)$ et 274 en sont exemptes $(\mathrm{BC}=0)$.

effets deviennent non significatifs quelle que soit la méthode utilisée.

En présence de bois de compression $(\mathrm{BC}=1$; $n=110$ ), ces deux méthodes fournissent par contre des résultats légèrement différents au niveau intraclonal : alors que les résidus des mesures réalisées par fréquence de résonance ne semblent pas influencés par ce facteur, ceux découlant des mesures par vitesse de l'onde ultrasonore en dépendent de manière significative.

Ces résultats tendent à démontrer l'absence d'interaction entre les méthodes de mesure du module d'élasticité et le facteur clone; en revanche, suivant le choix de la méthode de mesure, il peut apparaitre des différences de comportement entre ramets d'un même clone, dues à la méthode elle-même. Ici aussi, l'influence de la présence de bois de compression n'est pas à écarter.

\subsubsection{Effet de la position de l'éprouvette au sein de l'arbre}

La zone de prélèvement de chaque échantillon étant définie en fonction de la position du billon dans l'arbre et de l'orientation du plateau dont elle a été débitée, il est également possible d'évaluer l'impact de la position de 
Tableau VII. Influence des facteurs clone et ramet dans clone sur les résidus des droites de régression établies entre les mesures du module d'élasticité à l'aide de la méthode de référence et des deux méthodes acoustiques pour un ensemble de 384 éprouvettes de mélèze hybride. Influence de la présence/absence de bois de compression $(B C=1 / 0)$. Tableau synthétique des résultats d'analyse de la variance de type hiérarchisé.

\begin{tabular}{|c|c|c|c|c|}
\hline Méthode & Facteur & $\begin{array}{c}\text { Échantillon total } \\
n=384\end{array}$ & $\begin{array}{c}\text { significatio } \\
\mathrm{BC}=0 \\
n=274\end{array}$ & $\begin{array}{l}\mathrm{BC}=1 \\
n=110\end{array}$ \\
\hline
\end{tabular}

Tableau VIII. Influence des facteurs billon et orientation et leur interaction sur les résidus des droites de régression établies entre les mesures du module d'élasticité à l'aide de la méthode de référence et des deux méthodes acoustiques pour un ensemble de 384 éprouvettes de mélèze hybride. Tableau synthétique des résultats d'analyse de la variance de type croisé.

\begin{tabular}{lccc}
\hline Méthode & Facteur & \multicolumn{2}{c}{ Degré de liberté F } \\
\hline Fréquence de résonance & Billon & 2 & $5,53 * *$ \\
& Orientation & 3 & $0,49 \mathrm{~ns}$ \\
Vitesse de l'onde ultrasonore & Interaction & 6 & $0,72 \mathrm{~ns}$ \\
& Billon & 2 & $6,40 * *$ \\
& Orientation & 3 & $0,97 \mathrm{~ns}$ \\
& Interaction & 6 & $1,19 \mathrm{~ns}$ \\
\hline
\end{tabular}

l'éprouvette dans l'arbre sur la distribution des résidus via une analyse de la variance à deux facteurs. Le modèle testé se définit comme suit :

$$
Y_{i j k}=\mu+B_{i}+O_{j}+\left(B^{*} O\right)_{i j}+\varepsilon_{i j k}
$$

avec $Y_{i j k}=$ observation $\mathrm{k}$ du billon $\mathbf{i}$ et de l'orientation $\mathbf{j}$; $\mu=$ moyenne générale ;

$B_{i}=$ effet du billon i (facteur fixe) ;

$O_{j}=$ effet de l'orientation $\mathrm{j}$ (facteur fixe) ;

$\left(B^{*} O\right)_{i j}=$ interaction billon $\mathrm{i}$ - orientation $\mathbf{j}$; $\varepsilon_{i j k}=$ résidu lié au modèle d'analyse.
Cette analyse permet de mettre en évidence un effet hautement significatif du facteur billon, quelle que soit la méthode, alors que l'orientation et l'interaction de ces deux facteurs apparaissent non significatives (tableau VIII).

Sachant l'influence de la présence du bois de compression sur les résidus, mise en évidence plus haut, la surestimation globale des mesures au niveau du billon de pied (tableau IX) pourrait s'expliquer partiellement par la présence plus importante de ce bois de réaction à la base du tronc attestée par le tableau IX. Ceci confirme les travaux d'Yoshizawa et al. [26] sur Larix leptolepis.

\subsubsection{Effet de la masse volumique et de la largeur des cernes}

Sur la base de l'échantillon total, nous constatons l'existence d'une corrélation négative et très hautement significative entre la masse volumique et les résidus des deux droites de régression déduites des deux méthodes acoustiques (tableau $X$ ). Ces corrélations correspondent à une surestimation des modules d'élasticité dans les gammes de valeurs élevées. Il est donc vraisemblable que cette relation soit une nouvelle fois liée à la présence de bois de compression, caractéristique corrélée de manière positive à la masse volumique $[16,20]$. Cette hypothèse est renforcée par le calcul des coefficients de

Tableau IX. Résidus moyens (MPa) observés pour les 3 types de billons, sur la base des droites de régression calculées entre les valeurs du module d'élasticité établi par la méthode de référence et les deux méthodes acoustiques et pourcentage des éprouvettes présentant du bois de compression $(\mathrm{BC}=1)$.

\begin{tabular}{|c|c|c|c|}
\hline \multirow[b]{2}{*}{ Position du billon } & \multicolumn{2}{|c|}{ Résidus (MPa) } & \multirow[b]{2}{*}{$\%$ d'éprouvettes pour $\mathrm{BC}=1$} \\
\hline & Fréquence de résonance & Vitesse de l'onde ultrasonore & \\
\hline De pied & -68 & -173 & 39 \\
\hline Intermédiaire & 5 & 45 & 21 \\
\hline Supérieur & 63 & 128 & 26 \\
\hline
\end{tabular}


Tableau X. Coefficients de corrélation de Pearson $(R)$ entre la masse volumique et la largeur des cernes d'une part, et les résidus calculés sur la base des droites de régression établies entre les mesures du module d'élasticité par la méthode de référence et les deux méthodes acoustiques d'autre part, pour un ensemble de 384 éprouvettes de mélèze hybride dont 110 contiennent du bois de compression $(\mathrm{BC}=1)$ et 274 en sont exemptes $(\mathbf{B C}=0)$.

\begin{tabular}{|c|c|c|c|}
\hline \multirow[b]{2}{*}{ Méthode } & \multirow[b]{2}{*}{ Échantillon } & \multicolumn{2}{|c|}{$\mathrm{R}$} \\
\hline & & $\begin{array}{c}\text { Masse } \\
\text { volumique }\end{array}$ & $\begin{array}{c}\text { Largeur } \\
\text { des cernes }\end{array}$ \\
\hline $\begin{array}{l}\text { Fréquence de } \\
\text { résonance }\end{array}$ & $\begin{array}{c}n=384 \\
\mathrm{BC}=1 ; n=110 \\
\mathrm{BC}=0 ; n=274\end{array}$ & $\begin{array}{c}-0,26 * * * \\
-0,53 * * * \\
-0,18 * *\end{array}$ & $\begin{array}{c}0,06 \mathrm{~ns} \\
0,23 * \\
0,01 \mathrm{~ns}\end{array}$ \\
\hline $\begin{array}{l}\text { Vitesse de l'onde } \\
\text { ultrasonore }\end{array}$ & $\begin{array}{c}n=384 \\
\mathrm{BC}=1 ; n=110 \\
\mathrm{BC}=0 ; n=274\end{array}$ & $\begin{array}{c}-0,24 * * * \\
-0,51 * * * \\
-0,11 \mathrm{~ns}\end{array}$ & $\begin{array}{c}0,19 * * * \\
0,33 * * * \\
0,15 * *\end{array}$ \\
\hline
\end{tabular}

corrélation sur base de l'échantillon réduit aux 110 éprouvettes contenant du bois de compression, qui montrent une augmentation sensible de ces valeurs.

En revanche, en l'absence de ce défaut, ces coefficients de corrélation chutent, au point de devenir non significatifs dans le cas de la méthode ultrasonore tout en restant cependant significatifs dans le cas de la fréquence de résonance.

L'étude de l'influence de la largeur des cernes montre une corrélation positive de cette variable avec les résidus issus de l'équation établie à partir de la méthode ultrasonore. La présence de bois de compression semble également accentuer cette relation pour les deux méthodes testées ; cependant, dans le cas de la méthode ultrasonore, le coefficient de corrélation reste hautement significatif en l'absence de ce type de bois. Indépendamment de la présence de ce défaut, la largeur des cernes pourrait donc avoir une influence sur la distribution des résidus de la régression établie à partir des mesures effectuées par vitesse de l'onde ultrasonore, ce qui ne semble pas être le cas pour la méthode de la fréquence de résonance (tableau X).

\section{Conclusions et perspectives}

Par leur rapidité de mise en œuvre, tant la méthode de la mesure de la vitesse de l'onde ultrasonore (Sylvatest) que celle de la fréquence de résonance (Grindo-Sonic) présentent un avantage certain pour la détermination du module d'élasticité par rapport à la méthode de référence.
La valeur du module d'élasticité ne peut cependant pas s'obtenir directement mais bien via l'utilisation d'une équation de régression de type simple.

La dispersion des valeurs autour de ces droites de régression est faible, spécialement en ce qui concerne la méthode de la fréquence de résonance, et permet d'obtenir des mesures individuelles précises.

Il faut cependant être conscient que différentes caractéristiques physiques du bois peuvent provoquer des biais par rapport aux mesures réalisées par la méthode de référence $\left(\mathrm{MOE}_{4 \mathrm{PT}}\right)$. C'est principalement le cas de la présence de bois de compression, spécialement pour les valeurs obtenues à l'aide de la méthode ultrasonore. Pour pallier cet inconvénient, il y a donc lieu d'être particulièrement attentif au choix des éprouvettes afin d'éliminer ce bois de réaction avant toute mesure, et spécialement dans le cas du mélèze, d'éviter le pied du fût qui contient proportionnellement beaucoup de bois de compression.

Dans le cas de programmes d'amélioration génétique visant à augmenter la résistance mécanique du bois, ces deux méthodes semblent également utilisables. Elles seraient cependant beaucoup plus intéressantes si elles pouvaient fournir des informations fiables sur la base de mesures réalisées sur du matériel autre que des éprouvettes normalisées tel que des poutres en vraie grandeur, des billons, voire des arbres debout comme Fujisawa et al. [11] le laissent supposer en Cryptomeria. Dans ces conditions, d'autres caractéristiques telles que la nodosité, voire l'angle du fil, susceptibles d'influencer les résultats devraient être prises en compte. La sélection génétique pour les caractéristiques mécaniques du bois pourrait alors être réalisée à grande échelle comme cela peut se pratiquer pour les caractéristiques de croissance et certaines propriétés de base du bois comme la masse volumique.

Remerciements : Nous exprimons notre gratitude à Mme V. Bucur, Chargée de Recherche au Centre de Recherches forestières de Nancy, à Mr J. Hébert, Chef de Travaux à la Faculté universitaire des Sciences agronomiques de Gembloux, et Mr A. Nanson, Directeur au Centre de Recherche de la Nature, des Forêts et du Bois à Gembloux, pour avoir apporté leur critique constructive à la lecture de ce manuscrit.

Nous remercions aussi vivement tous les membres du personnel qui ont activement collaboré à cette étude, spécialement : Mme M. Passani, Mrs R. Buchet, A. Lemaire, T. Porphyre et M. Thielens. 


\section{Références}

[1] Anonyme, Norme belge ${ }^{\circ}$ NBN B 15-230, Essais des bétons, Essais non destructifs, Mesure de la Fréquence de résonance, $1976,8 \mathrm{p}$.

[2] Anonyme, Norme française NF-B 51-016. Bois, Essais de flexion statique. Détermination du module d'élasticité en flexion statique de petites éprouvettes sans défaut, 1987, 6 p.

[3] Baiilleres H., Calchera G., Demay L., Fouquet D., Vernay M., Classement des bois guyanais de structure. Comparaison de trois techniques non-destructives : visuelle, ultrasonore et analyse modale, in : $4^{\mathrm{e}}$ colloque sciences et industries du bois, Nancy, France, 11-13 septembre 1996, A.R.B.O.L.O.R. Nancy 1996, pp. 369-376.

[4] Bucur V., Détermination du module d'Young du bois par une méthode dynamique sur carottes de sondage, Ann. Sci. forest. 38 (1981) 283-298.

[5] Bucur V., Archer R.R., Elastic constants for wood by an ultrasonic method, Wood Sci. Technol. 18 (1984) 255-265.

[6] Chatre M., Gourlaouen J.C., Poirson G., Application de la détermination de la fréquence de résonance au contrôle des produits réfractaires, Conférence présentée au congrès de contrôle non-destructif à Mayence, avril 1978.

[7] Croiset G., Leclercq A., Lecomte H., Questienne P., Étude comparative de deux méthodes de détermination du module d'élasticité du bois et de ses dérivés, Station de Technologie forestière, CRA, Gembloux, Rapport interne, 1980, $52 \mathrm{p}$.

[8] Dagnelie P., Théorie et méthodes statistiques, Volume 2, Les Presses agronomiques de Gembloux ASBL, Belgique, 1986, 463 p.

[9] Dagnelie P., Statistique théorique et appliquée, Tome 1, Les Presses agronomiques de Gembloux ASBL, Belgique, 1992, $492 \mathrm{p}$.

[10] Dzbenski W., Versuche zur Anwendung der Ultraschall-Messtechnik bei der Gütesortierung von Konstruktionsholz nach seiner Festigkeit, Holzforschung und Holzverwertung 33 (1981) 105-1 10.

[11] Fujisawa Y., Ohta S., Nishimura K., Tajima M., Wood characteristics and genetic variations in sugi (Cryptomeria Japonica), clonal differences and correlations between locations of dynamic moduli of elasticity and diameter growths in plus-tree clones, Mokuzai Gakkaishi 38 (1992) 638-644.

[12] Görlacher R., Ein neues Messverfahren zur Bestimmung des Elastizitätsmoduls von Holz, Holz als Rohund Werkstoff 42 (1984) 219-222.
[13] Guillot J.L., Lanvin J.D., Sandoz J.L., Classement structure du sapin/épicéa par réseaux neuronaux à partir des mesures sylvatest, in : $4^{\mathrm{c}}$ colloque sciences et industries du bois, Nancy - France, $11-12-13$ septembre 1996, A.R.B.O.L.O.R. Nancy 1996, 377-384.

[14] Haines D.W., Leban J.M., Herbé C., Determination of Young's modulus for spruce, fir and isotropic materials by the resonance flexure method with comparisons to static flexure and other dynamic methods, Wood Sci. Technol. 30 (1996) 253-263.

[15] Haines D.W., Leban J.M., Evaluation of MOE of Norway spruce by the resonance flexure method, For. Prod. J. 47 (1997) 91-93.

[16] Jane F.W., The Structure of Wood, Adam \& Charles Black, London, 1956, 427 p.

[17] Jourez B., Leclercq A., Modelling Young's modulus on small clear specimens in relation to silvicultural treatment, Final report, Task 11, EEC Forest Project, n ${ }^{\circ}$ MA2B-0024 Gembloux, Belgique, 1994, 88 p.

[18] Kollmann F.F.P., Côté W.A., Principles of Wood Science and Technology, Volume 1: Solid Wood, SpringerVerlag, Berlin, 1984, $592 \mathrm{p}$.

[19] Lemmens J.X., Operating instructions for Grindo-Sonic MK3 equipment, Note technique, date non communiquée, $23 \mathrm{p}$.

[20] Low A.J., Compression wood in conifers, A review of literature, For. Abstr. 25 (1964) 9 p.

[21] Sandoz J.L., Grading of construction timber by ultrasound, Wood Sci. Technol. 23 (1989) 95-108.

[22] Sandoz J.L., Moisture content and temperature effect on ultrasound timber grading, Wood Sci. Technol. 27 (1993) 373-380.

[23] Sandoz J.L., Matériau, Architecture et Techniques, Ultrason et Bois de Construction, in : Construire en bois, Cours COMETT-Euroligna-EPFL-IBOIS, Suisse, 17 nov. 1994, 32 p.

[24] Spinner S., Tefft W.E., A method for determining mechanical resonance frequencies and for calculating elastic moduli from these frequencies, in : Proceedings ASTM Vol. 61, 1961, pp. 1221-1238.

[25] Timell T.E., Compression Wood in Gymnosperms, Volume 1, Springer-Verlag, Berlin, 1986, $706 \mathrm{p}$.

[26] Yoshizawa N., Okamoto Y., Idei T., Righting movement and xylem development in tilted young conifer trees, Wood Fiber Sci. 27 1986) 579-589. 